\title{
A cluster randomized controlled trial comparing Virtual Learning Collaborative and Technical Assistance strategies to implement an early palliative care program for patients with advanced cancer and their caregivers: a study protocol
}

Lisa Zubkoff ${ }^{12^{*}}$ (D), Kathleen Doyle Lyons ${ }^{3,4}$, J. Nicholas Dionne-Odom ${ }^{5,6,7}$, Gregory Hagley ${ }^{3}$, Maria Pisu ${ }^{1,7}$, Andres Azuero ${ }^{1,5,6}$, Marie Flannery ${ }^{8}$, Richard Taylor ${ }^{5,6}$, Elizabeth Carpenter-Song ${ }^{9}$, Supriya Mohile ${ }^{8 \dagger}$ and Marie Anne Bakitas $5,6,7+$

\begin{abstract}
Background: Virtual Learning Collaboratives (VLC), learning communities focused on a common purpose, are used frequently in healthcare settings to implement best practices. Yet, there is limited research testing the effectiveness of this approach compared to other implementation strategies. This study evaluates the effectiveness of a VLC compared to Technical Assistance (TA) among community oncology practices implementing ENABLE (Educate, Nurture, Advise, Before Life Ends), an evidence-based, early palliative care telehealth, psycho-educational intervention for patients with newly diagnosed advanced cancer and their caregivers.

Methods: Using Reach, Effectiveness, Adoption, Implementation, Maintenance (RE-AIM) and Proctor's Implementation Outcomes Frameworks, this two-arm hybrid type-III cluster-randomized controlled trial (RCT) will compare two implementation strategies, VLC versus TA, among the 48 National Cancer Institute Community Oncology Research Program (NCORP) practice clusters that have not historically provided palliative care to all patients with advanced cancer. Three cohorts of practice clusters will be randomized to the study arms. Each practice cluster will recruit 15-27 patients and a family caregiver to participate in ENABLE. The primary study outcome is ENABLE uptake (patient level), i.e., the proportion of eligible patients who complete the ENABLE program (receive a palliative care assessment and complete the six ENABLE sessions over 12 weeks). The secondary (Continued on next page)
\end{abstract}

\footnotetext{
* Correspondence: lisazubkoff@uabmc.edu

${ }^{\dagger}$ Supriya Mohile and Marie Anne Bakitas are co-senior authors

'Division of Preventive Medicine, Department of Medicine, University of Alabama at Birmingham, Birmingham, AL, USA

${ }^{2}$ Birmingham/Atlanta VA Geriatric Research Education and Clinical Center (GRECC), Department of Veterans Affairs, Birmingham, AL, USA

Full list of author information is available at the end of the article
}

(c) The Author(s). 2021 Open Access This article is licensed under a Creative Commons Attribution 4.0 International License, which permits use, sharing, adaptation, distribution and reproduction in any medium or format, as long as you give appropriate credit to the original author(s) and the source, provide a link to the Creative Commons licence, and indicate if changes were made. The images or other third party material in this article are included in the article's Creative Commons licence, unless indicated otherwise in a credit line to the material. If material is not included in the article's Creative Commons licence and your intended use is not permitted by statutory regulation or exceeds the permitted use, you will need to obtain permission directly from the copyright holder. To view a copy of this licence, visit http://creativecommons.org/licenses/by/4.0/. The Creative Commons Public Domain Dedication waiver (http://creativecommons.org/publicdomain/zero/1.0/) applies to the data made available in this article, unless otherwise stated in a credit line to the data. 
(Continued from previous page)

outcome is overall program implementation (practice cluster level), as measured by the General Organizational Index at baseline, 6, and 12 months. Exploratory aims assess patient and caregiver mood and quality of life outcomes at baseline, 12, and 24 weeks. Practice cluster randomization will seek to keep the proportion of rural practices, practice sizes, and minority patients seen within each practice balanced across the two study arms.

Discussion: This study will advance the field of implementation science by evaluating VLC effectiveness, a commonly used but understudied, implementation strategy. The study will advance the field of palliative care by building the capacity and infrastructure to implement an early palliative care program in community oncology practices.

Trial registration: Clinicaltrials.gov. NCT04062552; Pre-results. Registered: August 20, 2019. https://clinicaltrials.gov/ ct2/show/NCT04062552?term=NCT04062552\&draw=2\&rank=1

Keywords: Implementation strategies, Implementation science, Early palliative care, Advanced cancer, Clusterrandomized controlled trial

\section{Contributions to the literature}

- As recommended by oncology professional organizations, this study will promote the use of an evidence-based, telehealth, early palliative care program for patients with advanced cancer among a national sample of community oncology practices.

- Improve understanding of effectiveness of two commonly used implementation strategies, the Virtual Learning Collaborative (VLC) and Technical Assistance (TA).

- Evaluates measures of ENABLE implementation, organizational factors that influence fidelity to implementation, and patient and caregiver outcomes.

- Lessons learned will inform measurement of multi-level implementation outcomes related to early palliative care interventions.

- Lessons learned will inform future use of telehealth interventions and virtual implementation strategies.

\section{Introduction}

\section{Background and rationale}

Due to the benefits of early palliative care (EPC) in randomized controlled trials [1-3], the American Society of Clinical Oncology recommends " ... combined standard oncology care and palliative care ... early in the course of illness for any patient with metastatic cancer and/or high symptom burden" [4] (p.881). Yet, the National Cancer Institute (NCI)'s "Landscape Study" revealed that only $30 \%$ of community oncology practices have access to palliative care multidisciplinary teams in outpatient settings $[5,6]$. Even practices offering EPC are often unable to meet the needs of all advanced cancer patients due to a limited palliative care workforce and the large number of patients with advanced cancer and caregivers $[7,8]$. Training existing oncology staff to provide evidence-based palliative care interventions [9] is a potentially scalable and sustainable approach to address the national shortage of palliative care specialists. The ENABLE (Educate, Nurture, Advise, Before Life Ends) program is an example of such an intervention that can be implemented by healthcare organizations seeking to develop or enhance palliative care services.

ENABLE is an evidence-based, scalable model of EPC promoted by the National Cancer Institute EvidenceBased Cancer Control Programs (EBCCP), formerly known as Research-Tested Intervention Program (RTIP) [10-12]. ENABLE is led by a Nurse Coach and includes a comprehensive palliative care assessment, weekly telehealth coaching sessions (6 for patient; 3 for caregiver) using the Charting Your Course ${ }^{\circ}$ guidebook. The coaching sessions review essential palliative care topics, such as problem solving, coping, symptom management, self-care, communication, decision-making, and advance care planning and monthly follow-up calls (Fig. 1). The parallel ENABLE program topics for caregivers mirror the patient sessions. ENABLE has not been widely used in community oncology practices despite being publicly available through the EBCCP. An effective implementation strategy to address implementation barriers (e.g., lack of knowledge about evidence-based EPC, insufficient infrastructure) is needed to equip oncology practices to develop the skills to implement and sustain ENABLE. However, it is unclear which strategy would be most successful in assisting implementation.

Two common strategies to enhance implementation of evidence-based practices include VLC and TA. VLC is an innovative version of the traditional (face-to-face) Learning Collaborative, developed by the Institute for Healthcare Improvement (IHI) to guide clinical teams in implementing evidence-based practices [13]. Learning collaboratives use group interaction among teams to guide clinical improvement efforts. VLC, which uses a virtual (web-based) platform instead of face-to-face meetings, 


\begin{tabular}{|c|}
\hline$P A$ \\
\hline $\begin{array}{l}\text { Palliative Care Assessment following National Consensus Guidelines: } \\
\text { Understanding of illness, treatment plan, prognosis }>\text { Decision style and preferences }>\text { Goals of care }>\text { Physical } \\
\text { symptoms }>\text { Social history }>\text { Support system and challenges }>\text { Psycho-emotional well-being }>\text { Spirituality }>\text { Advance } \\
\text { care planning }>\text { Pharmacological assessment and recommendations }>\text { Referrals }>\text { Communicate with PCP }>\text { Follow-up }\end{array}$ \\
\hline $\begin{array}{l}\text { Chapter/Session 1: Problem Solving and the "COPE" Attitude } \\
\text { Distress Thermometer }>\text { What is problem solving coping? }>\text { COPE: A Positive Problem-Solving Attitude }>\text { "C"- } \\
\text { Creativity }>\text { "O"-Optimism }>\text { "P"-Planning }>\text { "E"-Expert Information }>\text { The Seven Steps of Problem-Solving }>\text { Identifying } \\
\text { the Problem }>\text { Establishing a Goal }>\text { Brainstorming Solutions }>\text { Pros and Cons }>\text { Picking and Implementing a Solution } \\
>\text { Reviewing how Your Plan Worked }>\text { ACTIVITY-Giving the } 7 \text { Steps a Shot }\end{array}$ \\
\hline $\begin{array}{l}\text { Chapter/Session 2: Self-Care } \\
\text { Distress Thermometer }>\text { What is Self-Care? }>\text { Healthy Eating and Nutrition }>\text { Exercise }>\text { Smoking }>\text { Relaxation } \\
\text { Techniques }>\text { ACTIVITY-Identifying a Support Team }>\text { ACTIVITY-Building a Support Team }>\text { What's the Latest? }\end{array}$ \\
\hline $\begin{array}{l}\text { Chapter/Session 3: Symptom Management } \\
>\text { Distress Thermometer }>\text { Prioritizing and Tracking Symptoms }>\text { Physical Symptoms of Cancer }>\text { Emotional Effects of } \\
\text { Cancer (Depression, Anxiety, Grief \& Loss) }>\text { ACTIVITY-Identifying Strengths and Resources }>\text { Spirituality }\end{array}$ \\
\hline $\begin{array}{l}\text { Chapter/Session 4: Core Values, Talking about what Matters Most, \& Making Decisions for the Future } \\
\text { >Distress Thermometer }>\text { Core Values and Decision-Making }>\text { ACTIVITY-Thinking About My Own Core Values }>\text { How } \\
\text { to Talk to Your Family and Health Care Team about What Matters Most to You }>\text { Decision Aids }>\text { ACTIVITY-Watching } \\
\text { the Looking Ahead DVD }>\text { ACTIVITY-The Ottawa Personal Decision Guide }>\text { Advance Directives }\end{array}$ \\
\hline $\begin{array}{l}\text { Chapter/Session 5: My Life Story } \\
\text { My Life Story and Why Thinking about it is Important (even if I'm not dying) }>\text { Where I was born and early childhood } \\
>\text { Influential people }>\text { Important events }>\text { Cherished times }>\text { Important goals }>\text { Favorite accomplishments/proudest } \\
\text { moments }>\text { Important elements if there was a movie about your life }\end{array}$ \\
\hline $\begin{array}{l}\text { Chapter/Session 6: Looking at Today, Looking at Tomorrow } \\
>\text { Distress Thermometer }>\text { What is Forgiveness }>\text { Regrets }>\text { People they want to forgive }>\text { Seeking forgiveness from } \\
\text { others }>\text { Things left undone }>\text { Feeling at peace }>\text { Family and personal values }>\text { Hopes and fears }>\text { Sources of strength } \\
>\text { Wisdom gained \& lessons learned }>\text { Advice for future generations }>\text { Future things to accomplish }\end{array}$ \\
\hline $\begin{array}{l}\text { Monthly Follow-up: } \\
\text { Ongoing psychosocial support }>\text { Reinforce/clarify previous material as it impacts real time decision making, symptom } \\
\text { management and QOL. }\end{array}$ \\
\hline CAREGIVER (CG) \\
\hline Chapter/Session 1: Mirrors PT Session 1 w \\
\hline Chapter/Session 2: Mirrors PT Session 2 \\
\hline Chapter/Session 3: Mirrors PT Session 3 with special attention to surrogate decision-making role \\
\hline Monthly Follow-up: Mirrors PT \\
\hline ollow-up: If PT \\
\hline
\end{tabular}

Fig. 1 Charting your course (CYC) chapter/session topics

was designed to decrease the cost of participation and increase the speed of incorporating lessons into practice [14, 15]. The evidence on VLCs appears to improve processes, but is inconclusive on outcomes $[16,17]$.

The second strategy, TA, is defined as "interactive support that is individualized to the specific needs of individuals or teams." (p.3) [18]. TA is typically provided one-on-one to a single organization and does not use a community structure to encourage shared learning or collaboration [19]. The evidence on TA effectiveness is minimal $[19,20]$. To our knowledge, there are few rigorous trials comparing the effectiveness of VLC or TA and more testing with rigorous study design is needed [14, $15,21,22]$. Which strategy would be most effective for the widespread implementation of ENABLE in community oncology practices is unclear.

To address these gaps in implementation science and palliative care, we developed a cluster randomized controlled trial (RCT) that compares VLC to TA for implementing ENABLE in community oncology practices. Our primary hypothesis is that practices randomized to VLC will outperform those randomized to TA resulting in a higher proportion of patients completing the ENABLE program. The more effective implementation of ENABLE will be due to the benefits of group dynamics (e.g., shared experiences, accountability, learning, discussion, and interactions), and quality improvement learning/skills that occur in the VLC but not in TA. Ultimately, a greater uptake of ENABLE will lead to better outcomes (mood and quality of life) for the patients and caregivers at the practices exposed to VLC compared to those exposed to TA.

\section{Study aims and hypotheses}

Based on Proctor's implementation framework, the purpose of this two-arm hybrid type-III cluster RCT in community oncology practices is to compare the effectiveness of two strategies, VLC and TA for implementing the evidence-based ENABLE program (Fig. 2).

\section{Primary aim (implementation outcome, patient level)}

Compare the effectiveness of VLC vs. TA on ENABLE program uptake, as measured by the proportion of patients who complete the ENABLE program (i.e., have a 


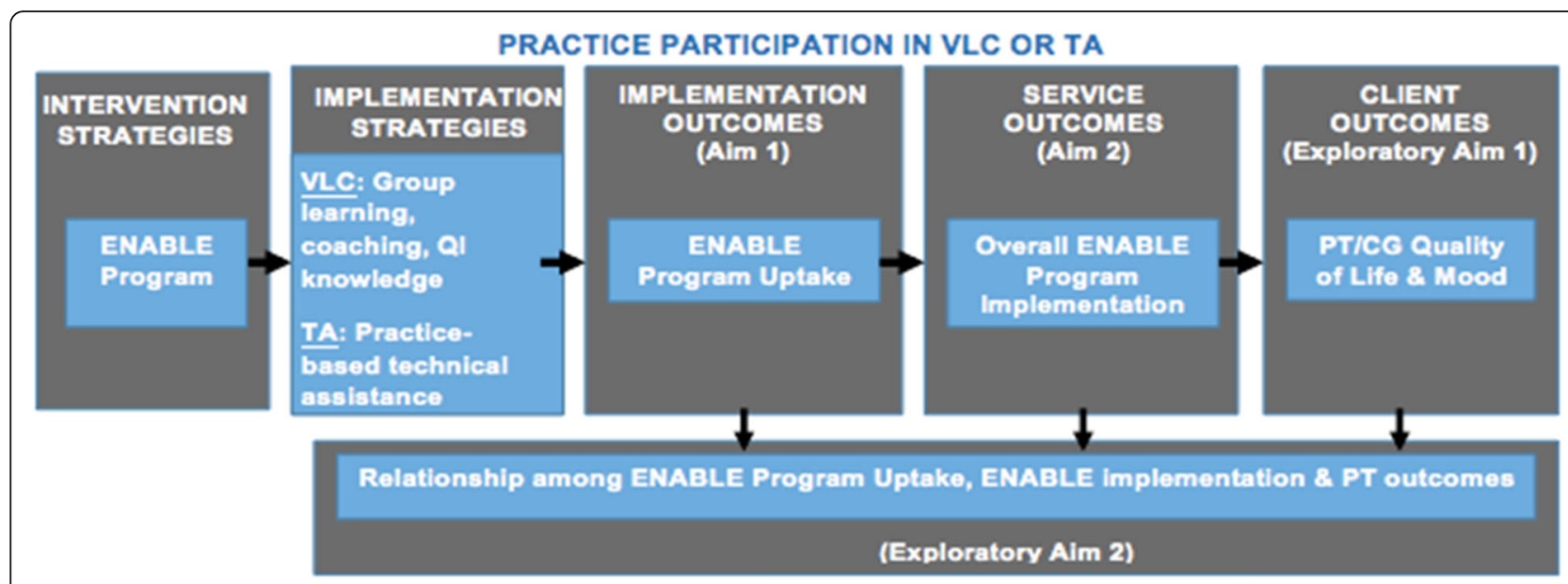

Fig. 2 Adapted proctor's implementation outcomes model applied to study aims

palliative care assessment and complete the six ENABLE sessions). Hypothesis: Practice clusters randomized to VLC will have greater ENABLE program uptake than practice clusters randomized to TA.

\section{Secondary aim (service outcome, practice level)}

Compare the effectiveness of VLC vs. TA on practice clusters' overall ENABLE program implementation. This will be assessed using the ENABLE General Organizational Index (GOI), which measures factors that influence institutional implementation of evidence-based practices. $\mathrm{Hy}$ pothesis: Practice clusters randomized to VLC will have better overall ENABLE program implementation than practice clusters randomized to TA.

\section{Exploratory aim 1 (patient \& caregiver outcomes)}

Compare practice clusters randomized to VLC or TA on patient and caregiver quality of life (QOL) and mood outcomes. Hypothesis: Patients and caregivers at practice clusters randomized to VLC will have better QOL and mood compared to those at practice clusters randomized to TA.

\section{Exploratory aim 2}

Determine the relationship among ENABLE program uptake, overall ENABLE program implementation, and patients' QOL and mood across the two strategies. Hypothesis: Practice clusters with better ENABLE program uptake and overall ENABLE implementation (regardless of implementation group) will demonstrate a higher degree of improved patient outcomes (i.e., QOL and mood) compared to practice clusters that have low/poor ENABLE program uptake and overall ENABLE implementation.

\section{Methods/design}

Theoretical framework

This study is guided by the Reach, Effectiveness, Adoption, Implementation, Maintenance (RE-AIM) framework, which is designed to enhance the quality, speed, and public health impact of efforts to translate research into "real world" settings [23, 24]. Further, this study applies Proctor's Implementation Outcomes framework [25] to assess implementation success via outcomes on three levels: implementation (effects of actions to implement new treatment), service (reflect the practice process changes), and client (evidence-based program's effect on the target population).

\section{Trial design}

This two-arm hybrid type-III cluster RCT compares implementation strategy effectiveness, while gathering additional information on the ENABLE program outcomes [26-28]. VLC and TA have distinct communication channels, time requirements, social systems, and structures $[29,30]$. A target sample of 48 NCI's Community Oncology Research Program (NCORP) practices will be randomized to one of two conditions: 1) VLC group which will receive ENABLE training and participate in a 15 -month VLC $(n=24)$ or 2$)$ TA group which will receive ENABLE training and participate in a 15-month TA process $(n=24)$. Each NCORP practice in this study will be formed into "practice clusters." Practice cluster is defined as an NCORP practice location where oncology physicians and study staff work independently and do not crossover to another practice. Practice clusters will be recruited in 3 cohorts of approximately 16 practice clusters per cohort. In each cohort half of the practice clusters will be randomized to VLC and half will be randomized to TA (Fig. 3). 


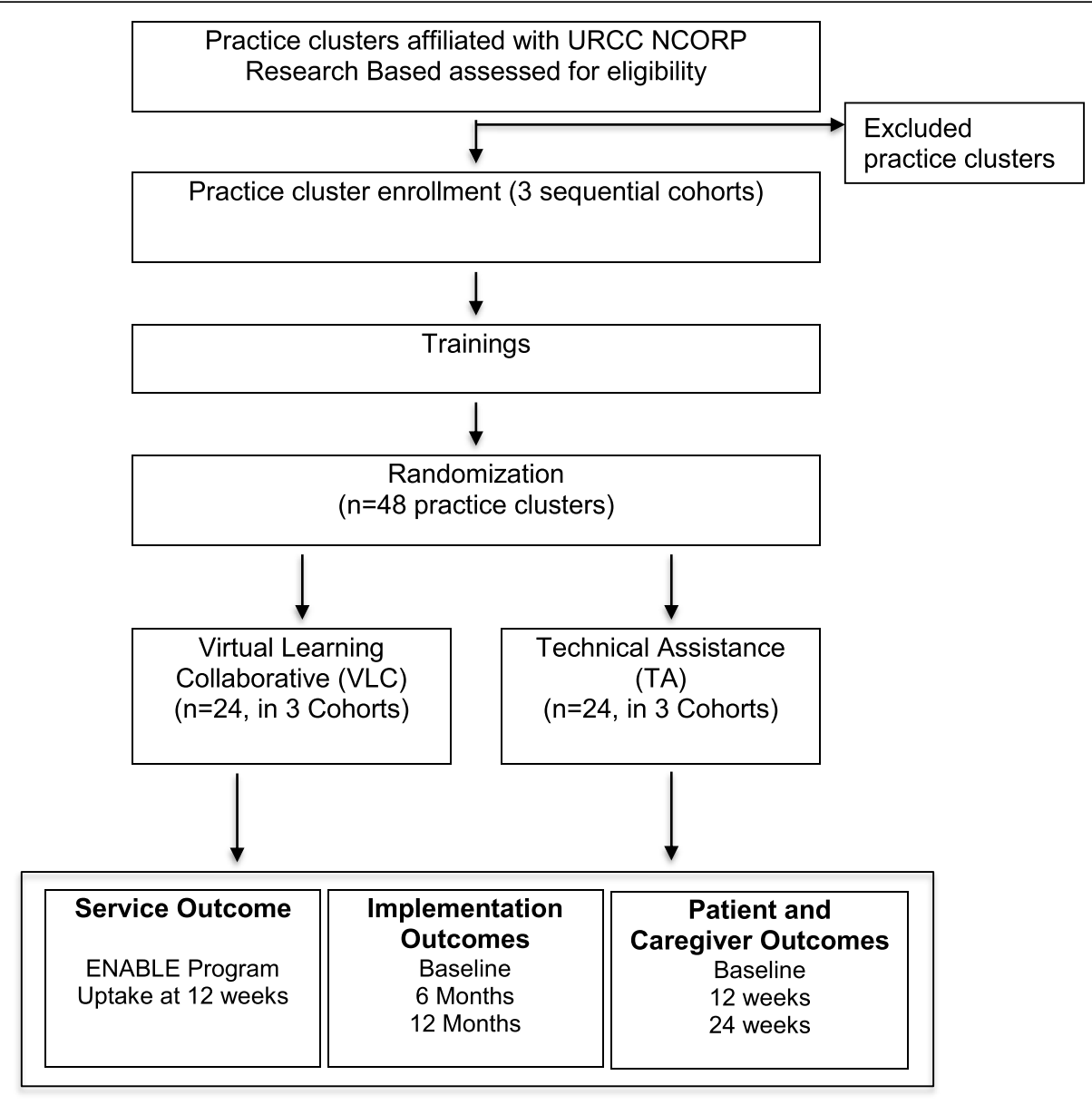

Abbreviations: URCC NCORP: University of Rochester Cancer Center NCI Community Oncology Research Program

Fig. 3 Trial design

\section{Setting/sample}

\section{URCC NCORP practice cluster participation}

This study is being carried out from the University of Rochester Cancer Center (URCC) NCORP Research Base. NCORP is a national network that brings cancer treatment and care delivery trials to people in community settings. NCORP is comprised of 7 Research Bases, and 46 Community Affiliates, each with affiliated practices [31]. The URCC NCORP Research Base is associated with 22 NCORPs and 358 sub-affiliates across the country. URCC NCORP practices vary in size and geographic location; however, to be eligible to participate in this study, they must meet the following inclusion criteria:

- Identify two (or more) Nurse Coaches to deliver the ENABLE program. For practices that only have 1 nurse available, one Nurse Coach is allowed.
- Commitment of Nurse Coaches to be trained to conduct the palliative care assessment and deliver the ENABLE sessions

- Desire to implement ENABLE, including presence of an investigator (e.g., Primary Affiliate Principal Investigator, oncology physician, Cancer Care Delivery Research (CCDR) Lead) and/or program administrator/supervisor who are willing to be key contacts

- Demonstrated support/buy-in from oncology physicians who are willing to enroll patients

- Agreement of practice leadership and staff to support/participate in study activities

- If necessary, willingness to participate in a phone interview to determine capacity to implement the ENABLE program.

Exclusion criteria Practice clusters that currently offer fully functioning inter-professional early palliative care 
to all cancer patients will be excluded. If practices offer early palliative care services to some, but not to all cancer patients, practices will only enroll patients to this study who have not received early palliative care services.

\section{Within practice cluster patients and caregivers participants \\ Patients}

Patients will be encouraged (but not required) to select a caregiver, defined below, to participate in the study. Participants can receive cancer treatment while participating in the study but will not have received palliative care services at the time of enrollment.

Patient inclusion criteria Patients must be diagnosed with advanced cancer (stage III/IV, recurrence, or progressive solid tumor cancer) within the last 90 days and have an expected survival of at least 6 months. Patients must be English-speaking, at least 18 years old, willing to complete the palliative care assessment and ENABLE sessions, have access to a telephone that can receive calls, and able to provide informed consent to participate in the study.

Patient exclusion criteria Patients who received previous palliative care services will be excluded. However, after enrollment, patients may receive palliative care services outside of the ENABLE program, if appropriate.

\section{Caregivers}

Each patient can identify a single caregiver to participate in the study. The caregiver is any person who provides support to the patient. Caregivers may remain in the study even if the patient withdraws.

Caregiver inclusion criteria Caregivers are defined as an "unpaid relative or friend who knows them well and who provides regular support to their cancer." Caregivers must be English-speaking, at least 18 years old, willing to complete the ENABLE Caregiver program sessions, have access to a telephone that can receive calls, and able to provide informed consent to participate in the study.

\section{Exclusion criteria None.}

\section{Practice cluster recruitment}

The URCC NCORP Research Base will announce the study via email to all URCC NCORP community affiliates and post study activation on the Cancer Trials Support Unit (CTSU) website. In addition, we will hold information webinars. Interested practice clusters will complete a form to describe the practice (e.g., current palliative care services, demographics of patient population, staff members to be involved in the study) and submit it to URCC Research Base. Each practice cluster will be expected to enroll and deliver ENABLE to 15-27 patients.

\section{Patient and caregiver recruitment}

Staff in each practice cluster will identify eligible patients from clinical schedules and inform oncology physicians. If the physician determines the patient to be eligible and the patient agrees to be informed about the study, study personnel at the practice (e.g., Nurse Coach, research coordinator) will meet the patient either in person or via HIPAA compliant phone or videoconference to review the study. Informed consent will be obtained either in person or using a HIPAA compliant phone or videoconference and documented.

\section{Evidence-based practice to be implemented: ENABLE} The ENABLE program for patients consists of [1] a standardized in-person or telehealth Palliative Care Assessment [32] and [2] a series of six, 20-60 min weekly telephone coaching sessions provided by a Nurse Coach using the Charting Your Course $\odot$ (CYC) guidebook [33, 34]. ENABLE fosters patient empowerment $[35,36]$ through coaching on problem-solving coping, symptom management, self-care, communication, decisionmaking, and life review and reflection. A Nurse Coach, who is a registered nurse or advanced practice provider (e.g., nurse practitioner or physician assistant) who has at least 2 years' experience working in oncology or palliative care, delivers the ENABLE program. Caregivers receive a separate series of similar telephone sessions using a CYC@ guidebook with caregiver-relevant content. After completing the weekly sessions, both the patient and caregiver receive monthly check-in phone calls. Figure 1 presents the CYC@ session topics. Trials testing ENABLE have demonstrated improved patient quality of life, mood, and survival outcomes and reduced caregiver burden and improved mood [10, 11, 37].

\section{Implementation interventions (practice cluster level)}

Table 1 summarizes the essential elements of the VLC and TA implementation strategies and outlines the distinctive features between the two. Each practice cluster will assemble an implementation team who will participate in the assigned implementation strategy (VLC or TA). At minimum, this includes one or two Nurse Coaches who will deliver the intervention and at least one coordinator who will facilitate recruitment and patient and caregiver outcome assessment. Other members of the practice cluster (e.g., oncologists, data managers) are also encouraged to participate in implementation team and VLC or TA activities. 
Table 1 Summary of Implementation Strategies

\begin{tabular}{lll}
\hline Activity & Virtual Learning Collaborative (VLC) & Technical Assistance (TA) \\
\hline Form an ENABLE Implementation Team & $\checkmark$ & $\checkmark$ \\
ENABLE training & $\checkmark$ & $\checkmark$ \\
ENABLE Implementation (15 months) & $\begin{array}{l}\text { Group web-based VLC learning sessions } \\
\text { (monthly) }\end{array}$ & $\begin{array}{l}\text { Individual practice cluster consultation call with ENABLE } \\
\text { expert (monthly) }\end{array}$ \\
Coaching/Expert Consultation & $\checkmark$ & $\checkmark$ \\
Quality Improvement Knowledge and & $\checkmark$ & \\
Information & & \\
Individualized Written Feedback & $\checkmark$ & \\
Monthly Group Discussion and Sharing & $\checkmark$ & \\
Between Session Activities & $\checkmark$ & \\
\hline
\end{tabular}

\section{Virtual Learning Collaborative}

Practice clusters randomized to the 15-month VLC will participate in monthly virtual group-based learning sessions to discuss key components of the ENABLE program, implementation progress (e.g., nurse coach training, number referrals, number patients/caregivers enrolled, sessions completed), data collection activities, and current successes and challenges. In addition, the VLC will include instruction on quality improvement tools and techniques to assist with the implementation process, coaching, and the opportunity to share stories and experiences and learn from other practice clusters using an "all teach, all learn" approach [13]. The VLC will be co-led by ENABLE and quality improvement experts (RT and KDL).

\section{TA}

Practice clusters randomized to the 15-month TA study arm will receive individual, interactive monthly consultation calls with an ENABLE expert (JND-O). The TA leader will ask similar questions about the practice cluster's implementation progress as are covered in the VLC study arm (e.g., nurse training, referral process, enrollment, sessions completed, data collection activities, and current successes and challenges). The relationship between the TA provider (JND-O) and practice clusters will be friendly, trusting, strengths-based, collaborative, and will support practice cluster autonomy. We anticipate that practice clusters will solicit input or guidance from the TA leader on challenges or areas of concern. The TA leader will offer advice and suggestions to the degree desired by the practice cluster.

\section{Study trainings}

Nurse Coaches who deliver the ENABLE program will complete the approximately 19 -h self-paced 10 -module ENABLE training on a web-based platform (Canvas). The training, developed by ENABLE experts, consists of [1] an overview of the ENABLE program; [2] instruction on how to conduct the palliative care assessment and deliver each ENABLE session; [3] role play of the assessment and phone sessions that will be reviewed by an ENABLE expert who will provide feedback; and [4] tools and resources for Nurse Coaches. Upon completion of the ENABLE training, Nurse Coaches will complete an evaluation of the training to receive Continuing Education Unit (CEU) credits.

Additional trainings will cover study procedures and how to use the web-based study platforms: Canvas, Zoom, and Research Electronic Data Capture (REDCap), an electronic data capture tool to enter study data.

\section{Measures and outcomes}

Based on Proctor's Framework (Table 2), this study has 3 categories of outcomes [1] implementation (aim 1), [2] service (aim 2), and [3] client (patient and caregiver) outcomes (exploratory aim 1 ).

\section{Aim 1: ENABLE uptake (implementation outcome, patient level)}

The primary outcome will be ENABLE program uptake (program completion). To capture ENABLE program uptake, each ENABLE Nurse Coach will use a standardized ENABLE Nurse Coach Contact Log to document patient and caregiver referrals, enrollment, and completion of the essential ENABLE elements (i.e., palliative care assessment, ENABLE sessions). The Nurse Coach Contact Log, which is completed in REDCap, will document each contact and ENABLE session using an assigned study identification for each patient or caregiver.

\section{Aim 2: Overall ENABLE Program implementation (service outcome, practice cluster level)}

This outcome will be measured for each practice cluster via phone interviews with Nurse Coaches and coordinators using the ENABLE General Organizational Index (GOI). This instrument was developed to assess factors that influence institutional implementation of the ENABLE program [38]. We previously adapted [38] the 
Table 2 Measures and outcomes

\begin{tabular}{lllll}
\hline Outcome & Instrument(s) & $\begin{array}{l}\text { Time } \\
\text { Point }\end{array}$ & Target & Description \\
\hline Aim 1 Outcome & (Implementation Outcome, Patient Level) & & \\
ENABLE Uptake & ENABLE Nurse Coach Contact & Months & Coordinator or Nurse & $\begin{array}{l}\text { This contact log documents the delivery of each } \\
\end{array}$ \\
& Log (patients and caregivers) & $1-15$ & Coach & ENABLE session.
\end{tabular}

\section{Aim 2 Outcome (Service Outcome, Practice Level)}

Overall Program ENABLE GOI

Implementation
Baseline, Coordinator and Nurse 6,12 Coach (other staff may months attend call)
The ENABLE General Organizational Index (GOI) is conducted via a phone interview with the Coordinator and Nurse Coach (other staff may attend call) to assess factors that influence institutional implementation and fidelity to an evidence-based practice. It covers 12 domains and is scored on a Likert scale. Qualitative analysis will also be conducted [38-40].

Additional Implementation Outcomes

ENABLE Program ENABLE RE-AIM Self-Assessment Implementation Tool

Baseline, Coordinator and Nurse
6,12 Coach
months

Perceptions of Provider Perceptions of Early Early Palliative Care Palliative Care Survey

Baseline, 15 Oncologist and Nurse 5 Coach

months

\section{VLC and TA Process Outcomes} VLC Practice Participation and 1-15 TA lead)
Program Participation and Engagement (VLC Engagement; TA Practice Participation and Engagement

Months Study team (VLC lead,

Months Study team (VLC lead, 1-15 TA lead)

ENABLE Process Measures

Palliative Care Assessment

Palliative Care Assessment Checklist

Additional ENABLE Contacts
ENABLE Additional Contacts Log Months Coordinator and Nurse (patients and caregivers)
The ENABLE RE-AIM Self Assessment Tool (Reach, Efficiency, Adoption, Implementation, and Maintenance) was developed in our prior work to collect reach, adoption, implementation, and maintenance [23, 24, 38]. Reach is defined as the number of enrolled participants divided by the number of eligible participants. Efficacy will be measured by patient and caregiver quality of life and mood outcomes (see Explanatory Aim 1 measures). Adoption will be measured by calculating the number of oncology physicians who refer patients divided by those who have the opportunity to refer patients to the study. Implementation is captured in the study's primary outcome, ENABLE uptake. Maintenance will be measured by the presence of the ENABLE program 6 months after the completion of the VLC or TA implementation strategy in the ENABLE Sustainment Survey. The ENABLE Nurse Coach and/or coordinator will complete the online ENABLE RE-AIM Self-Assessment tool at baseline, 6 months ( \pm 8 weeks), and 1 year ( \pm 8 weeks).

The survey asks about attitudes about palliative care, feelings about palliative care specialists, comfort with an preferences for providing early concurrent oncology palliative care, and description of early concurrent palliative care provided in the practice cluster $[38,41]$. Responses are on a Likert scale.

The VLC and TA Participation and Engagement forms assess the extent to which the sites participate in the assigned strategy (VLC or TA). The forms share commonalities, but are distinct to reflect the different methods of communicating with practice clusters. The site's level of participation in the session is scored on a Likert scale with an additional free text entry. A higher score indicates better participation and engagement.

The instrument measures fidelity and adherence to key features of the VLC and TA study arms respectively. The forms will be completed after each session. The instrument for the VLC and TA study arm are distinct. The study team will audit 10\% of sessions by reviewing the recording and verifying key components being discussed during sessions.

This form documents each of the key components of the palliative care assessment that are completed.

This instrument tracks other patient and caregiver contacts or activities as part of the ENABLE program. The ENABLE Nurse Coach will document non-session contacts and activities each week (i.e., time spent doing ENABLE related study tasks other than delivering the 
Table 2 Measures and outcomes (Continued)

\begin{tabular}{|c|c|c|c|c|}
\hline Outcome & Instrument(s) & $\begin{array}{l}\text { Time } \\
\text { Point }\end{array}$ & Target & Description \\
\hline
\end{tabular}

Fidelity to Palliative Palliative Care Assessment Care Assessment Adjudication Checklist

Sustainment

ENABLE Sustainment Survey

Month

21

Baseline, Nurse Coach

months

$4-6$

Oncologist and Nurse

Coach (may be staff)

Baseline, Patient

12,24

weeks

Baseline, Patient

12,24

weeks

Additional Patient Outcomes

Patient Global PROMIS Global Health

Health

Symptom burden

MDASI

Baseline, Patient

12,24

weeks

Baseline, Patient completed by other

sessions) and enter them into REDCap. The coordinator will also document the time to complete recruitment and enrollment procedures in the log on REDCap every 2 weeks.

An ENABLE expert will review four of each practice cluster's palliative care assessments to ensure completion and correct documentation. At each practice cluster, we will request the clinical note in the medical record associated with the palliative care assessment for the first two patients enrolled and then a random sample of two additional patients. Study personnel will review the note and compare it to the Palliative Care Assessment Checklist. Adequate fidelity will be demonstrated by $\geq 85 \%$ of the components identified on the Palliative Care Assessment Checklist.

This 9-item instrument measures the presence/sustainment of the ENABLE program 6 months ( \pm 4 weeks) after completion of the 15-month implementation strategy (VLC or TA). Example questions include the number of trained ENABLE Nurse Coaches, number of patient and caregiver participants, ENABLE components currently offered, and any adaptations that have been made to the ENABLE program. Other staff members (e.g., coordinator, Primary Affiliate PI, CCDR Lead) may also complete the survey.

The Hospital Anxiety and Depression Scale (HADS) instrument assesses mood, including anxiety and depression. Seven questions rate the depression subscale and 7 questions rate the anxiety subscale. Each item has a 4-point scale, ranging from 0 to 3 with possible scores ranging from 0-21 for each subscale. Scoring for each sub-scale is as follows: 0-7 Normal, 8-10 Borderline abnormal, and 11-21 Abnormal [42-46].

The FACIT-Pal consists of the FACT-G (Functional Assessment of Cancer Therapy-General), a general measure of quality of life, and the palliative care subscale (Pal), which assesses issues specifically relevant to palliative care $[37,47-49]$. The FACT-G is a 27-item questionnaire that provides a total score as well as four subscale scores: physical, social/family, emotional, and functional wellbeing. The FACIT-Pal includes 19 additional concerns relevant for persons at the end of life. The total score is the sum of the FACT-G plus the FACIT-Pal subscale [50].

12,24

weeks

The 10-item measure uses Likert-scale response options for each item, ranging from 1 (always) to 5 (never). This instrument produces 2 scores: physical health and mental health score [51-54].

The MD Anderson Symptom Inventory (MDASI) is a 19item measure that assesses symptom severity experienced by patients with cancer and the symptom-related interference with daily living $[55,56]$. The MDASI uses a 0-10 severity scale for 13 items, with 0 being symptom "not present" and 10 being "the worst you can imagine," and an interference 0-10 response scale for 6 items ranging from 0 being "did not interfere" to 10 being "interfered completely".

Patients will be asked to report their education level, marital status, whom they live with, employment status, age, gender, ethnicity, race, employment status, diagnosis, insurance status, annual household income,
Baseline Patient
Demographic and
Clinical

Characteristics 
Table 2 Measures and outcomes (Continued)

\begin{tabular}{|c|c|c|c|c|}
\hline Outcome & Instrument(s) & $\begin{array}{l}\text { Time } \\
\text { Point }\end{array}$ & Target & Description \\
\hline & & & & $\begin{array}{l}\text { urban vs. rural living location, religious preference, } \\
\text { presence of a living companion, prior and current } \\
\text { smoking status and usage, prior and current alcohol } \\
\text { consumption. }\end{array}$ \\
\hline \multicolumn{5}{|c|}{ Exploratory Aim 1 Outcome (Caregiver Outcomes) } \\
\hline Caregiver Mood & HADS & $\begin{array}{l}\text { Baseline, } \\
12,24 \\
\text { weeks }\end{array}$ & Caregiver & See description under Patient Outcomes. \\
\hline $\begin{array}{l}\text { Caregiver Quality } \\
\text { of Life }\end{array}$ & PROMIS Global Health & $\begin{array}{l}\text { Baseline, } \\
12,24 \\
\text { weeks }\end{array}$ & Caregiver & See description under Patient Outcomes. \\
\hline Caregiver burden & $M B C B$ & $\begin{array}{l}\text { Baseline, } \\
12,24 \\
\text { weeks }\end{array}$ & Caregiver & $\begin{array}{l}\text { The Montgomery Borgatta Caregiver Burden (MBCB) } \\
\text { Scale is a 14-item instrument assesses caregiver burden } \\
\text { and the impacts on the caregiver's life [57-59]. Each } \\
\text { item uses a Likert-type scale response option, ranging } \\
\text { from } 1 \text { ("A lot less") to } 5 \text { ("A lot more"). }\end{array}$ \\
\hline $\begin{array}{l}\text { Positive Aspects of } \\
\text { Caregiving }\end{array}$ & Positive Aspects of Caregiver & $\begin{array}{l}\text { Baseline, } \\
12,24 \\
\text { weeks }\end{array}$ & Caregiver & $\begin{array}{l}\text { The Positive Aspects of Caregiver is a 9-item instrument } \\
\text { that assesses positive aspects of caregiving, including } \\
\text { the extent to which the caregiver feels: useful, good } \\
\text { about him/herself, needed, appreciated, important, } \\
\text { strong and confident, appreciates life, more positive atti- } \\
\text { tude toward life, and strengthened relationships with } \\
\text { others. Each item is rated on a } 5 \text {-point ordinal scale ran- } \\
\text { ging from } 1 \text { (disagree a lot) to } 5 \text { (agree a lot) [60]. }\end{array}$ \\
\hline $\begin{array}{l}\text { Preparedness for } \\
\text { Caregiving }\end{array}$ & $\begin{array}{l}\text { Preparedness for Caregiving } \\
\text { Scale }\end{array}$ & $\begin{array}{l}\text { Baseline, } \\
12,24 \\
\text { weeks }\end{array}$ & Caregiver & $\begin{array}{l}\text { The Preparedness for Caregiving Scale is a self-rated in- } \\
\text { strument that consists of 8-items that asks caregivers } \\
\text { about their perceived preparedness for multiple do- } \\
\text { mains of caregiving, such as providing physical care, } \\
\text { providing emotional support, setting up in-home sup- } \\
\text { port services, and dealing with the stress of caregiving } \\
\text { [61, 62]. Responses are rated on a 5-point scale with } \\
\text { scores ranging from } 0 \text { (not at all prepared) to } 4 \text { (very } \\
\text { well prepared) [63]. There is also a question with a free } \\
\text { text response. }\end{array}$ \\
\hline Demographics & Demographic Form & Baseline & Caregiver & $\begin{array}{l}\text { Caregivers will also be asked to report education level, } \\
\text { marital status, whom they live with, employment status, } \\
\text { age, gender, ethnicity, race, employment status, } \\
\text { insurance status, annual household income, urban vs. } \\
\text { rural living location, religious preference, relationship to } \\
\text { patient participant, if the patient participants lives with } \\
\text { them, average number of days per week they help take } \\
\text { care of the patient, and average hours per day helping } \\
\text { the patient participant. }\end{array}$ \\
\hline
\end{tabular}

General Organizational Index (GOI) which measures fidelity to an evidence-based practice by assessing two key elements of implementation: individualization and quality improvement [64]. The GOI has been shown to have acceptable psychometric properties [39]. The ENABLE GOI structured interview guide covers 12 domains (program philosophy, eligibility/client identification, penetration, assessment, individualized initial palliative care treatment plan, individualized subsequent ENABLE contacts, supervision, process monitoring, outcome monitoring, quality assurance, and client choices regarding service provision) associated with successful implementation. ENABLE GOI assessments will occur at baseline, 6 months ( \pm 8 weeks), and 1 year ( \pm 8 weeks). After the phone interview, the interviewer [40] will score each domain on a Likert scale for a total range of 12-60. Moreover, interviews will be recorded and transcribed for qualitative analysis to augment quantitative assessment of this outcome.

Table 2 presents additional implementation measures, including RE-AIM, process outcomes for the study arms (VLC and TA), and ENABLE process measures.

\section{Exploratory aim: patient measures}

Table 2 presents patient measures. Patient assessments will be completed at baseline, 12 , and 24 weeks. There will be several options to collect the data: [1] study coordinator reads the questions to the patient (either in 
person or on the phone) and enters the patient responses, [2] the patient completes the assessments in person with a study coordinator, or [3] the patient will take a paper copy home, complete it, and return it at either the next visit, via mail, or scan and email.

\section{Exploratory aim: Caregiver measures}

Caregiver assessments (Table 2) will be completed at baseline, 12 and 24 weeks using the same methods as for patients described above.

\section{Sample size}

\section{Aim 1: Implementation outcome (patient level)}

The RCT is powered for the aim 1 outcome, ENABLE program uptake, defined as the proportion of patients who complete the ENABLE program. In determining the targeted sample size, we made the following assumptions: [1] an intra-class correlation coefficient (ICC) value of 0.05 , [2] alpha of $1 \%(\alpha=0.01)$ and $80 \%$ power, [3] 20\% enrolled patients for whom completion of ENABLE cannot be assessed due to death or becoming too ill to continue with participation, and [4] a proportion of patients completing ENABLE of 0.52 in prior studies. With 24 practice clusters per group and a minimum of 12 patients per practice cluster (down from 15 after $20 \%$ unable to assess completion), for a total of 288 patients per group, the detectable difference in ENABLE program uptake is $0.18(\mathrm{OR}=2.06$; $\mathrm{RR}=1.33)$, a medium effect size.

\section{Randomization}

This cluster-randomized trial will be carried out in 3 sequential cohorts of $8-20$ practice clusters $(4-10$ per study arm) to reach the desired sample size of 48 practice clusters. Within each cohort, RCT arm assignment will occur after completion of all required study trainings, including the ENABLE training (Fig. 3). A minimization algorithm for group assignment with random initial start will be used to attempt to simultaneously balance the proportion of rural practices, practice sizes, and minority patients seen within each practice across the two RCT arms. The minimization algorithm will be generated using $\mathrm{R}$ software. Study team members and practice cluster members will not be blinded to group assignment or study hypotheses.

\section{Statistical analysis}

All randomized practice clusters will be included in primary comparisons, according to their group assignment, and regardless of their degree of participation in the study. Primary data analysis will include descriptive statistics for baseline practice cluster characteristics, patient characteristics, and outcomes in each RCT arm. We will examine the balance between RCT arms with respect to baseline characteristics (e.g., age, sex, race, cancer type, stage) using effect sizes, such Cohen's d and Cramer's. Factors showing non-trivial imbalances between comparators will be used as adjusting covariates in the between-group comparisons. Analysis will be conducted using the latest versions of SAS and R.

\section{Analysis for aim 1: Implementation outcome (patient level)}

We will use a logit GEE model with exchangeable correlation structure (to account for clustering within practice) for the binary patient uptake indictor ("yes" if the patient completed a palliative care assessment and all ENABLE program sessions prior to the 12 week assessments and "no" if the patient is still alive but has not completed a palliative care assessment and all six ENABLE program sessions prior to the 12-week assessments), with the group assignment as the main predictor. Model-predicted outcome proportions, odds ratios, relative risks, and confidence intervals for these measures will be used for interpretation. Supplemental analyses using appropriate methods for clustered data will consist of between-group comparisons of the proportion of recruited patients who could not complete the ENABLE sessions due to illness or death, and the number of sessions completed among all patients recruited.

\section{Analysis for aim 2: Service outcome (practice cluster level)} Analysis will compare VLC vs. TA on NCORP practices' overall ENABLE program implementation measured by the ENABLE GOI. Due to the small sample size for this practice cluster-level outcome (24 VLC vs. 24 TA), rather than conducting formal inference testing, comparisons will be descriptive statistics and effect size measures.

Qualitative analysis The audio-recorded interviews of the ENABLE GOI will be coded by study team members (EC-S) [40] in Atlas.ti using a qualitative content analysis approach using $a$ priori domains from the GOI measure and additional categories that emerge from the inductive review of transcripts [65]. Coded data will be aggregated and reviewed within and across codes to construct an in-depth understanding of facilitators, barriers, and contextual factors that may influence implementation of the ENABLE program.

Findings from the qualitative data will be integrated with the main findings of the RCT at the interpretation and reporting stage [66]. We will use a convergent mixed methods analysis to collect both qualitative and quantitative ENABLE GOI data at roughly the same time, assess information using parallel constructs for both types of data, separately analyze both types of data, and then compare results through discussion and jointly 
displaying both forms of data. The joint display will map each ENABLE GOI domain score to corresponding concepts or themes from these qualitative findings to more fully understand the context of the GOI domain scores and identify patterns across practice clusters [67]. This will provide validation for each data type and create a foundation for drawing conclusions about the implementation of the ENABLE program [68].

\section{Analysis for exploratory aims (patient and caregiver outcomes)}

Exploratory aim 1 To compare NCORP practices randomized to VLC or TA on patient and caregiver quality of life and mood, linear mixed models will be used to estimate differences in patient and caregiver outcome trajectories over the 24-week follow-up period. If necessary, a false discovery rate adjustment will be used to adjust for multiple exploratory inferences [69].

Exploratory aim 2 The association between ENABLE sessions and change in patient outcomes will be examined with linear mixed models. Adjusting covariates will include patient characteristics that may influence participation in sessions (e.g., sex, illness acuity, age). Linear mixed models will also be used to examine the association between GOI scores and patient outcomes.

\section{Discussion}

The relative strengths of learning collaboratives compared to other implementation strategies are poorly understood [16, 17]. This study will provide a direct comparison between the VLC and TA strategies to determine if VLC is a superior method to facilitate implementation of the evidence-based ENABLE program in community-based oncology practices. Finding successful strategies to implement models of early palliative care in cancer is imperative given national guidelines that have highlighted this priority [70].

Most published studies on VLCs are descriptive and have not definitively tested its effectiveness to enhance adoption and implementation [16, 71-82]. There are also few randomized-clinical trials that evaluate traditional in-person learning collaboratives [14, 15, 21, 22] or compare their effectiveness to other implementation approaches $[16,17,71,83]$. For example, learning collaboratives have been shown to outperform financial incentives or toolkits, but only on some process or secondary outcomes [16, 17]. This study will contribute to the fields of implementation science and palliative care as we will learn about the implementation effectiveness of VLC and TA while delivering an evidence-based practice. While we hypothesize the VLC will outperform TA due to the shared wisdom generated by the group, the VLC strategy has greater demands for sites and facilitators (and possibly costs) and has less opportinuity for individual attention than TA. As such, it is important to assess the added value of each strategy in order to know how to better spread and sustain EPC. Therefore, this study will inform efforts to more widely disseminate ENABLE, while simultaneously advancing implementation science methods and approaches that can be used for other evidence-based practices.

Like most research, we have encountered challenges during the study start-up period. First, the COVID-19 pandemic significantly impacted the ability for NCORP practices to conduct research and resulted in a need to rely on telehealth. Although most ENABLE activities were already offered via telephone, we modified the protocol so that all study procedures can now be conducted remotely, including the consent process and the comprehensive palliative care assessment. During this time, NCORP practices also expressed concern about the time required to complete the Nurse Coach training. To reduce training burden, the length of the web-based ENABLE Nurse Coach training was reduced from $25 \mathrm{~h}$ to approximately $19 \mathrm{~h}$. We anticipate the reduced number of training hours may be beneficial beyond the study given our goal to make the ENABLE training more broadly scalable to nurses and advanced practice providers interested in implementing this EPC program. We also amended the originally required cohort size $(n=16)$ and will permit cohorts of $8-$ 20 practice clusters (4-10 per study arm). To account for this in analyses, we will assess for confounding by cohort. Lastly, although each practice cluster will be expected to deliver ENABLE to 15-27 patients, we recognize that may not happen. To account for this, we will include an option to recruit additional practice clusters (up to $20 \%$ of 48 which is 58 practices) and additional cohorts. Despite these challenges, we anticipate the first cohort will launch in the first half of 2021.

There are several anticipated lessons learned from this study. First, to the best of our knowledge, this is the first large-scale study measuring the implementation of early palliative care in community-based oncology practices. To measure implementation, we developed and refined palliative care implementation measures and anticipate that lessons learned will be used to improve these measures. Second, this study not only seeks to identify the superior implementation strategy (VLC or TA), but also to examine the key components of each strategy. To do so, we have developed measures of participation, engagement, and fidelity that have not been applied in other published literature. Lessons learned will inform refinement of the measures and advance the field.

\section{Strengths and limitations}

There are several strengths to this study. First, this study will evaluate both the uptake of an evidence-based EPC 
intervention and organizational factors associated with improving delivery of EPC in community based care. Second is the collaboration with the URCC NCORP. Conducting this work outside a consortium would be difficult, particularly with respect to recruiting practice clusters. Third, this study provides a solution to the limited palliative care workforce by implementing this program as a generalist palliative care model [84]. As part of study activities, Nurse Coaches will be trained on how to deliver the ENABLE EPC program, thereby increasing practice capacity to offer palliative care services $[7,8$, 85]. A final, yet unanticipated strength to this study is the opportunity to implement the effective ENABLE EPC telehealth model in community oncology clinics during COVID-19 when telehealth services are particularly important.

While this study will contribute to implementation science methods and approaches, there are limitations. Patients and caregivers will not know which study arm their practice cluster is assigned. However, practice clusters will not be blinded to study aims and hypotheses as sharing those are essential to study recruitment and consent procedures. Similarly, blinding the TA and VLC facilitators and the assessors (e.g., ENABLE GOI interviewer) to the group assignment is not feasible. However, it is feasible to limit their knowledge of specific activities in each study arm to minimize bias. Contamination between practice cluster cohorts is another potential limitation. Since all practice clusters will be affiliated with URCC NCORP, enrolled practice clusters may discuss their study involvement with practices that register for a future cohort. To minimize potential contamination between cohorts, we will instruct second and third cohorts to reach out to the TA or VLC members (depending on randomization assignment) as opposed to outside practices for advice.

\section{Conclusion}

The results from this study will be twofold: [1] we will learn about the implementation of an early palliative care intervention among community oncology practices, and [2] we will learn about the effectiveness of the VLC as compared to TA to facilitate such implementation. In addition, lessons learned will advance the field of implementation science through further refinement of palliative care implementation measures and of the VLC implementation strategy.

\footnotetext{
Abbreviations

ACS: American Cancer Society; CCDR: Cancer Care Delivery Research; CCM: Chronic Care Model; CIC: Chronic Illness Care; CIRB: Central Institutional Review Board; CONSORT: Consolidated standards of reporting trials; CYC: Charting Your Course; DSMC: Data Safety Monitoring Committee; EBP: Evidence-based practice; ENABLE: Educate, Nurture, Advise, Before Life Ends; EPC: Early Palliative Care; ERIC: Expert Recommendations for Implementing Change; FACT-G: Functional Assessment of Cancer Therapy-
}

General; FACIT-PAL: Functional Assessment of Chronic Illness-Palliative Care; GOI: General Organizational Index; HADS: Hospital Anxiety and Depression Scale; ICC: Intra-class Correlation Coefficient; LC: Learning Collaborative; BMCB: Montgomery Borgatta Caregiver Burden Scale; MDASI: MD Anderson Symptom Inventory; NCl: National Cancer Institute; NCORP: NCI Community Oncology Research program; PI: Principal investigator; PROMIS: Patient Reported Outcome Measurement Information System; QOL: Quality of life; RCT: Randomized controlled trial; RE-AIM: Reach, Effectiveness, Adoption, Implementation, Maintenance Framework; REDCap: Research Electronic Data Capture; RUCA: Rural-Urban Commuting Area; TA: Technical Assistance; UAB: University of Alabama at Birmingham; UR: University of Rochester; URCC: University of Rochester Cancer Center; VLC: Virtual Learning Collaborative

\section{Acknowledgements}

The research reported in this protocol uses resources of the Dartmouth Hitchcock Medical Center, Dartmouth College, The Norris Cotton Cancer Center, University of Alabama, at Birmingham, University of Rochester, University of Rochester Cancer Center, and University of Rochester Cancer Center NCORP Research Base. Some work for this manuscript was conducted while Dr. Zubkoff was affiliated with the Geisel School of Medicine at Dartmouth, Dartmouth Hitchcock Medical Center, and the White River Junction VA Medical Center.

\section{Trial status}

The study is actively recruiting practices for cohort 1 .

Protocol amendments

Protocol amendments are reviewed and approved by NCI CIRB.

\section{Informed consent}

Current, state, federal, and institutional regulations concerning informed consent will be followed. Patients must have capacity to sign an informed consent. Consent documents will be signed by patients and caregivers; copies of the signed documents will be provided to the participants. A waiver of documentation of consent will be used for oncology physicians, ENABLE Nurse Coaches, Coordinators, and Other NCORP staff (e.g., Primary Affiliate PIs, CCDR lead, administrators/supervisors).

\section{Confidentiality}

All written materials will be kept confidential, locked in the private offices, and limited-access file room of the URCC NCORP Research Base and identified by ID numbers. All electronic information will be kept confidential with password-protected, limited access.

\section{Access to data}

Per protocol, all study data will be retained by the URCC NCORP Research Base for 5 years after completion of the study. Designated research personnel, including those at Dartmouth and UAB, is granted access to the data stored in the REDCap and Access databases. After completion of the study and publication of the study results, the de-identified study data will be available on the NCTN/NCORP Data Archive.

\section{Data and safety monitoring plan}

The James P Wilmot Cancer Center Data Safety Monitoring Committee (DSMC) will serve as the DSMC of record for this study. The DSMC provides oversight of study progress and safety by review of accrual and events at regularly scheduled meetings. The DSMC will monitor all adverse event rates. DSMC reports will be available for download on the Research Base website.

\section{Provenance and peer review}

Not commissioned; peer-reviewed for ethical and funding approval prior to submission.

\section{Disclaimer}

The funder did not have a role in the design or conduct of the study, and the authors have ultimate authority over study activities. The content is solely the responsibility of the authors and does not necessarily represent the official views of the National Cancer Institute. 


\section{Authors' contributions}

$L Z, M A B$, and $S M$ conceived the study and $L Z, M A B, S M$, JND-O, AA, MP, MF, and $\mathrm{KL}$ contributed to the study design. $\mathrm{LZ}, \mathrm{KL}, \mathrm{JND}-\mathrm{O}, \mathrm{GH}, \mathrm{MP}, \mathrm{AA}, \mathrm{MF}, \mathrm{RT}$, EC-S, SM, and MAB drafted the manuscript, and all authors provided comments and feedback for improvement the manuscript. All authors approved the final version of the manuscript and are responsible for their contributions.

\section{Funding}

Research reported in this publication was supported by the National Cancer Institute of the National Institutes of Health under Award Number R01CA229197. The content is solely the responsibility of the authors and does not necessarily represent the official views of the National Institutes of health.

\section{Availability of data and materials}

The investigators will provide access to data according to $\mathrm{NIH}$ and University of Rochester NCl Community Oncology Research Program policies.

\section{Ethics approval and consent to participate}

This study, URCC18110CD, was reviewed and approved by the NCI Central Institutional Review Board (CIRB). Original approval was received on July 2 , 2019. Subsequent amendments were approved on October 11, 2019, September 3, 2020, and October 21, 2020. NCI CIRB acts as the single IRB of record for all study sites. The protocol is registered at ClinicalTrials.gov (NCT04062552).

\section{Consent for publication}

Not applicable.

\section{Competing interests}

The authors declare that they have no competing interests.

\section{Author details}

'Division of Preventive Medicine, Department of Medicine, University of Alabama at Birmingham, Birmingham, AL, USA. ${ }^{2}$ Birmingham/Atlanta VA Geriatric Research Education and Clinical Center (GRECC), Department of Veterans Affairs, Birmingham, AL, USA. ${ }^{3}$ Dartmouth Hitchcock Medical Center, Lebanon, NH, USA. ${ }^{4}$ Department of Psychiatry, Geisel School of Medicine, Hanover, NH, USA. ${ }^{5}$ School of Nursing, University of Alabama at Birmingham, Birmingham, AL, USA. 'Division of Gerontology, Geriatrics and Palliative Care, UAB Center for Palliative and Supportive Care, Department of Medicine, University of Alabama at Birmingham, Birmingham, AL, USA. ${ }^{7} \mathrm{O}^{\prime}$ Neal Comprehensive Cancer Center, Birmingham, AL, USA. ${ }^{8}$ University of Rochester Medical Center, Rochester, NY, USA. ${ }^{9}$ Department of Anthropology, Dartmouth College, Hanover, NH, USA.

\section{Received: 13 January 2021 Accepted: 26 January 2021}

\section{Published online: 11 March 2021}

\section{References}

1. Kavalieratos D, Corbelli J, Zhang D, Dionne-Odom JN, Ernecoff NC, Hanmer J, et al. Association between palliative care and patient and caregiver outcomes: a systematic review and meta-analysis. JAMA. 2016;316(20):210414.

2. Meneses K, Landier W, Dionne-Odom JN. Vulnerable population challenges in the transformation of cancer care. Semin Oncol Nurs. 2016:32(2):144-53.

3. Hoerger M, Wayser GR, Schwing G, Suzuki A, Perry LM. Impact of interdisciplinary outpatient specialty palliative care on survial and quality of life in adults with advanced cancer: a meta-analysis of randomized controlled trials. Ann Behav Med. 2018;53(7):674-85.

4. Smith TJ, Temin S, Alesi ER, Abernethy AP, Balboni TA, Basch EM, et al. American Society of Clinical Oncology provisional clinical opinion: the integration of palliative care into standard oncology care. J Clin Oncol. 2012; 30(8):880-7

5. Carlos RC, Sicks JD, Chang GJ, Lyss AP, Stewart TL, Sung L, et al. Capacity for cancer care delivery research in National Cancer Institute Community Oncology Research Program Community Practices: availability of radiology and primary care research partners. J Am Coll Radiol. 2017;14(12):1530-7.
6. Kent EE, Mitchell SA, Castro KM, DeWalt DA, Kaluzny AD, Hautala JA, et al. Cancer care delivery research: building the evidence base to support practice change in community oncology. J Clin Oncol. 2015;33(24):2705-11.

7. Kamal AH, Wolf SP, Troy J, Leff V, Dahlin C, Rotella JD, et al. Policy changes key to promoting sustainability and growth of the specialty palliative care workforce. Health Aff (Millwood). 2019;38(6):910-8.

8. Lupu D, Quigley L, Mehfoud N, Salsberg ES. The growing demand for hospice and palliative medicine physicians: will the supply keep up? J Pain Symptom Manage. 2018;55(4):1216-23.

9. Hui D, Bruera E. Models of palliative care delivery for patients with cancer. J Clin Oncol. 2020;38(9):852-65.

10. Bakitas M, Lyons KD, Hegel MT, Balan S, Brokaw FC, Seville J, et al. Effects of a palliative care intervention on clinical outcomes in patients with advanced cancer: the Project ENABLE II randomized controlled trial. JAMA 2009:302(7):741-9.

11. Bakitas MA, Tosteson TD, Li Z, Lyons KD, Hull JG, Li Z, et al. Early versus delayed initiation of concurrent palliative oncology care: patient outcomes in the ENABLE III randomized controlled trial. J Clin Oncol. 2015;33(13): 1438-45.

12. Dionne-Odom JN, Azuero A, Lyons KD, Hull JG, Tosteson T, Li Z, et al. Benefits of early versus delayed palliative care to informal family caregivers of patients with advanced cancer: outcomes from the ENABLE III randomized controlled trial. J Clin Oncol. 2015;33(13):1446-52.

13. The breakthrough series: IHI's collaborative model for achieving breakthrough improvement. Boston, MA; 2003.

14. Nadeem E, Olin SS, Hill LC, Hoagwood KE, Horwitz SM. Understanding the components of quality improvement collaboratives: a systematic literature review Milbank O 2013:91(2):354-94.

15. Schouten LM, Hulscher ME, van Everdingen JJ, Huijsman R, Grol RP. Evidence for the impact of quality improvement collaboratives: systematic review. BMJ. 2008:336(7659):1491-4

16. Fu LY, Zook K, Gingold JA, Gillespie CW, Briccetti C, Cora-Bramble D, et al. Strategies for improving vaccine delivery: a cluster-randomized trial. Pediatrics. 2016:137(6)

17. Speroff T, Ely EW, Greevy R, Weinger MB, Talbot TR, Wall RJ, et al. Quality improvement projects targeting health care-associated infections: comparing virtual collaborative and toolkit approaches. J Hosp Med. 2011;6(5):271-8.

18. Leeman J, Calancie L, Hartman MA, Escoffery CT, Herrmann AK, Tague LE, et al. What strategies are used to build practitioners' capacity to implement community-based interventions and are they effective?: a systematic review. Implement Sci. 2015;10:80

19. Chilenski SM, Perkins DF, Olson J, Hoffman L, Feinberg ME, Greenberg M, et al. The power of a collaborative relationship between technical assistance providers and community prevention teams: a correlational and longitudinal study. Eval Program Plann. 2016:54:19-29.

20. Katz J, A W. Technical assistance to enhance prevention capacity: a research synthesis of the evidence base. Prev Sci. 2016;17(4):417-28.

21. Hulscher ME, Schouten LM, Grol RP, Buchan H. Determinants of success of quality improvement collaboratives: what does the literature show? BMJ Qual Saf. 2013;22(1):19-31

22. Nadeem E, Olin S, Hill L, Hoagwood K, Horwitz S. A literature review of learning collaboratives in mental health care: used but untested. Psychiatr Serv. 2014:65(9):1088-99.

23. What is RE-AIM? 2016 [Available from: http://www.re-aim.org

24. Glasgow RE, McKay HG, Piette JD, Reynolds KD. The RE-AIM framework for evaluating interventions: what can it tell us about approaches to chronic illness management? Patient Educ Couns. 2001:44(2):119-27.

25. Proctor $E$, Silmere $H$, Raghavan $R$, Hovmand $P$, Aarons $G$, Bunger $A$, et al. Outcomes for implementation research: conceptual distinctions, measurement challenges, and research agenda. Adm Policy Ment Health. 2011;38(2):65-76.

26. Curran GM, Bauer M, Mittman B, Pyne JM, Stetler C. Effectivenessimplementation hybrid designs: combining elements of clinical effectiveness and implementation research to enhance public health impact. Med Care. 2012;50(3):217-26

27. Damschroder LJ, Moin T, Datta SK, Reardon CM, Steinle N, Weinreb J, et al. Implementation and evaluation of the VA DPP clinical demonstration: protocol for a multi-site non-randomized hybrid effectivenessimplementation type III trial. Implement Sci. 2015;10:68.

28. Smelson DA, Chinman M, McCarthy S, Hannah G, Sawh L, Glickman M. A cluster randomized Hybrid Type III trial testing an implementation support 
strategy to facilitate the use of an evidence-based practice in VA homeless programs. Implement Sci. 2015;10:79.

29. Dearing JW, Kreuter MW. Designing for diffusion: how can we increase uptake of cancer communication innovations? Patient Educ Couns. 2010; 81(Suppl):S100-10.

30. Rogers EM. Diffusion of innovations. Fifth Edition ed. New York: Free Press; 2003.

31. National Cancer Institute. Community Oncology Research Program. US: Department of Health and Human Services; 2020. [Available from: https:// ncorp.cancer.gov/about/.

32. Manca DP, Aubrey-Bassler K, Kandola K, Aguilar C, Campbell-Scherer D, Sopcak $N$, et al. Implementing and evaluating a program to facilitate chronic disease prevention and screening in primary care: a mixed methods program evaluation. Implement Sci. 2014;9:135.

33. Wagner EH, Austin BT, Davis C, Hindmarsh M, Schaefer J, Bonomi A. Improving chronic illness care: translating evidence into action. Health Aff (Millwood). 2001;20(6):64-78.

34. Wasson JH, Splaine ME, Bazos D, Fisher ES. Overview: working inside, outside, and side by side to improve the quality of health care. Jt Comm J Qual Improv. 1998;24(10):513-7.

35. McCorkle R, Ercolano E, Lazenby M, Schulman-Green D, Schilling LS, Lorig K, et al. Self-management: enabling and empowering patients living with cancer as a chronic illness. CA Cancer J Clin. 2011;61(1):50-62.

36. Dionne-Odom JN, Kono A, Frost J, Jackson L, Ellis D, Ahmed A, et al. Translating and testing the ENABLE: CHF-PC concurrent palliative care model for older adults with heart failure and their family caregivers. J Palliat Med. 2014;17(9):995-1004.

37. Bakitas M, Lyons KD, Hegel MT, Balan S, Barnett KN, Brokaw FC, et al. The project ENABLE II randomized controlled trial to improve palliative care for rural patients with advanced cancer: baseline findings, methodological challenges, and solutions. Palliat Support Care. 2009;7(1):75-86.

38. Zubkoff L, Dionne-Odom JN, Pisu M, Babu D, Akyar I, Smith T, et al. Developing a "toolkit" to measure implementation of concurrent palliative care in rural community cancer centers. Palliat Support Care. 2017:1-13.

39. Heiervang KS, Egeland KM, Landers M, Ruud T, Joa I, Drake RE, et al. Psychometric properties of the general organizational index (GOI): a measure of individualization and quality improvement to complement program fidelity. Adm Policy Ment Health. 2020.

40. Freund T, Wensing M, Mahler C, Gensichen J, Erler A, Beyer M, et al. Development of a primary care-based complex care management intervention for chronically ill patients at high risk for hospitalization: a study protocol. Implementation Science. 2010;5(1):70.

41. Babu D, Dionne-Odom JN, Smith T, Zubkoff L, Bakitas M. Oncology providers' perceptions of early/concurrent palliative care. In: Annual Assembly of the Americal Academy of Hospice and Palliative Medicine and the Hospice and Palliative Nurses Association. Chicago: IL; 2016.

42. Bjelland I, Dahl AA, Haug TT, Neckelmann D. The validity of the hospital anxiety and depression scale. An updated literature review. J Psychosom Res. 2002;52(2):69-77.

43. Clark A, Fallowfield $\sqcup$. Quality of life measurements in patients with malignant disease: a review. J R Soc Med. 1986;79(3):165-9.

44. Herrmann C. International experiences with the hospital anxiety and depression scale--a review of validation data and clinical results. J Psychosom Res. 1997;42(1):17-41.

45. McMillan SC, Small BJ, Weitzner M, Schonwetter R, Tittle M, Moody L, et al. Impact of coping skills intervention with family caregivers of hospice patients with cancer: a randomized clinical trial. Cancer. 2006;106(1):214-22.

46. Zigmond AS, Snaith RP. The hospital anxiety and depression scale. Acta Psychiatr Scand. 1983;67(6):361-70.

47. Brady MJ, Cella D. Assessing quality of life in palliative care. Cancer Treat Res. 1999;100:203-16.

48. Brady MJ, Cella DF, Mo F, Bonomi AE, Tulsky DS, Lloyd SR, et al. Reliability and validity of the functional assessment of cancer therapy-breast qualityof-life instrument. J Clin Oncol. 1997;15(3):974-86.

49. Lyons KD, Bakitas M, Hegel MT, Hanscom B, Hull J, Ahles TA. Reliability and validity of the functional assessment of chronic illness therapy-palliative care (FACIT-Pal) scale. J Pain Symptom Manage. 2009;37(1):23-32.

50. Facit.org. Functional Assessment of Chronic Illness Therapy- Palliative Care. 2020. [Available from https:/www.facit.org/measures/FACIT-Pal]. Accessed 22 Feb 2020.

51. Cella D, Riley W, Stone A, Rothrock N, Reeve B, Yount S, et al. The PatientReported Outcomes Measurement Information System (PROMIS) developed and tested its first wave of adult self-reported health outcome item banks: 2005-2008. J Clin Epidemiol. 2010;63(11):1179-94.

52. Hays RD, Bjorner JB, Revicki DA, Spritzer KL, Cella D. Development of physical and mental health summary scores from the patient-reported outcomes measurement information system (PROMIS) global items. Qual Life Res. 2009;18(7):873-80.

53. HealthMeasures. Obtain \& Administer Measures: Northwestern University; 2018 [Available from: http://www.healthmeasures.net/explore-measurementsystems/promis/obtain-administer-measures.

54. HealthMeasures. Global Health: Northwestern University; 2018 [Available from: http://www.healthmeasures.net/administrator/components/com_ instruments/uploads/PROMIS Global Scoring Manual.pdf.

55. Center MAC. The MD Anderson Symptom Inventory: University of Texas; 2018 [Available from: https://www.mdanderson.org/research/departmentslabs-institutes/departments-divisions/symptom-research/symptomassessment-tools/md-anderson-symptom-inventory.html.

56. Cleeland CS, Mendoza TR, Wang XS, Chou C, Harle MT, Morrissey M, et al. Assessing symptom distress in cancer patients: the M.D. Anderson Symptom Inventory. Cancer. 2000;89(7):1634-46.

57. Montgomery R. Using and interpreting the montgomery borgatta caregiver burden scale 2016 [Available from: https://www.researchgate.net/profile/ Rhonda_Montgomery2/publication/265679222_Using_and_Interpreting_ the_Montgomery_Borgatta_Caregiving_Burden_Scale/links/56aac20508aed5 a01358c5bd.pdf?origin=publication_list.

58. Montgomery R, Borgatta E, Borgatta M. Societal and family change in the burden of care. In: Liu W, Kendig H, editors. Who Should Care for the Elderly? Singapore: Singapore University Press; 2000. p. 27-54.

59. O'Hara RE, Hull JG, Lyons KD, Bakitas M, Hegel MT, Li Z, et al. Impact on caregiver burden of a patient-focused palliative care intervention for patients with advanced cancer. Palliat Support Care. 2010;8(4):395-404.

60. Tarlow BJ, Wisniewski SR, Belle SH, Rupert M, Ory MG, Gallagher-Thompson D. Positive aspects of caregiving: contributions of the REACH project to the development of new measures for alzheimer's caregiving. Research on Aging. 2004;26(4):429-53.

61. Archbold PG, Stewart BJ, Greenlick MR, Harvath T. Mutuality and preparedness as predictors of caregiver role strain. Res Nurs Health. 1990;13(6):375-84.

62. Carter JH, Stewart BJ, Archbold PG, Inoue I, Jaglin J, Lannon M, et al. Living with a person who has Parkinson's disease: the spouse's perspective by stage of disease. Parkinson's Study Group. Mov Disord. 1998;13(1):20-8.

63. Zwicker D. Preparedness for Caregiving Scale. New York: New York University, College of Nursing; 2010.

64. McHugo GJ, Drake RE, Whitley R, Bond GR, Campbell K, Rapp CA, et al. Fidelity outcomes in the National Implementing Evidence-Based Practices Project. Psyciatr Serv. 2007;58(10):1279-84

65. Brooks J, McCluskey S, Turley E, King N. The utility of template analysis in qualitative psychology research. Qual Res Psychol. 2015;12(2):202-22.

66. Fetters MD, Curry LA, Creswell JW. Achieving integration in mixed methods designs-principles and practices. Health Serv Res. 2013;48(6 Pt 2):2134-56.

67. Guetterman TC, Fetters MD, Creswell JW. Integrating quantitative and qualitative results in health science mixed methods research through joint displays. Ann Fam Med. 2015;13(6):554-61.

68. Wisdom J, Creswell JW. Mixed methods: integrating quantitative and qualitative data collection and analysis while studying patient-centered medical home models. Rockville, MD; 2013 February 2013. Report No:: 13-0028-EF.

69. Glickman ME, Rao SR, Schultz MR. False discovery rate control is a recommended alternative to Bonferroni-type adjustments in health studies. J Clin Epidemiol. 2014;67(8):850-7.

70. Ferrell BR, Temel JS, Temin S, Alesi ER, Balboni TA, Basch EM, et al. Integration of Palliative Care Into Standard Oncology Care: American Society of Clinical Oncology Clinical Practice Guideline Update. J Clin Oncol. 2017;35(1):96-112.

71. Boushon B, Provost L, Gagnon J, Carver P. Using a virtual breakthrough series collaborative to improve access in primary care. Jt Comm J Qual Patient Saf. 2006:32(10):573-84

72. Butler A, Canamucio A, Macpherson D, Skoko J, True G. Primary care staff perspectives on a virtual learning collaborative to support medical home implementation. J Gen Intern Med. 2014;29(Suppl 2):S579-88.

73. Cranley LA, Norton PG, Cummings GG, Barnard D, Estabrooks CA. SCOPE: safer care for older persons (in residential) environments: a study protocol. Implement Sci. 2011;6:71.

74. Gance-Cleveland B, Aldrich H, Dandreaux D, Oetzel KB, Schmiege S. A virtual childhood obesity collaborative: satisfaction with online continuing education. J Pediatr Health Care. 2015;29(5):413-23. 
75. Gance-Cleveland B, Aldrich H, Schmiege S, Tyler K. Virtual obesity collaborative with and without decision-support technology. Int I Qual Health Care. 2016;28(3):316-23.

76. Gingold JA, Briccetti C, Zook K, Gillespie CW, Gubernick RS, Moon RY, et al. Context matters: practitioner perspectives on immunization delivery quality improvement efforts. Clin Pediatr (Phila). 2016;55(9):825-37.

77. John T, Morton M, Weissman M, O'Brien E, Hamburger E, Hancock Y, et al. Feasibility of a virtual learning collaborative to implement an obesity Q project in 29 pediatric practices. Int J Qual Health Care. 2014;26(2):205-13.

78. McHugh MC, Margolis LH, Rosenberg A, Humphreys E. Advancing MCH interdisciplinary/interprofessional leadership training and practice through a learning collaborative. Matern Child Health J. 2016;20(11):2247-53.

79. Zubkoff L, Neily J, Mills PD, Borzecki A, Shin M, Lynn MM, Gunnar W, Rosen A. Using a virtual breakthrough series collaborative to reduce postoperative respiratory failure in 16 Veterans Health Administration hospitals. Jt Comm J Qual Patient Saf. 2014;40(1):11-20.

80. Zubkoff L, Neily J, King B, Dellefield ME, Krein S, Young-Xu Y, Boar S, Mills PD. Virtual breakthrough series, part 1: preventing catheter-associated urinary tract infection and hospital-acquired pressure ulcers in the veterans health administration. Jt Comm J Qual Patient Saf. 2016;42(11):485-98.

81. Zubkoff L, Neily J, Quigley P, Soncrant C, Young-Xu Y, Boar S, Mills PD. Virtual breakthrough series, part 2: improving fall prevention practices in the veterans health administration. Jt Comm J Qual Patient Saf. 2016;42(11):497517.

82. Zubkoff L, Neily J, King B, Morgan S, Young-Xu Y, Boar S, Mills PD. Preventing pressure ulcers in the VHA using a virtual breakthrough series. J Nurs Care Qual. 2016;32(4):301-8.

83. Aschbrenner K.A., Pratt S.I, Bond GR., Zubkoff L, Naslund J.A., Jue K, et al. A virtual learning collaborative to implement health promotion in routine mental health settings: protocol for a cluster randomized trial. Contemp Clin Trials. 2019;84(105816)

84. Quill TE, Abernethy AP. Generalist plus specialist palliative care--creating a more sustainable model. N Eng J Med. 2013;368(23465068):1173-5.

85. Lupu D. American Academy of Hospice and Palliative Medicine Workforce Task Force. Estimate of current hospice and palliative medicine physician workforce shortage. J Pain Symptom Manage. 2010;40(6):899-911.

\section{Publisher's Note}

Springer Nature remains neutral with regard to jurisdictional claims in published maps and institutional affiliations.

Ready to submit your research? Choose BMC and benefit from:

- fast, convenient online submission

- thorough peer review by experienced researchers in your field

- rapid publication on acceptance

- support for research data, including large and complex data types

- gold Open Access which fosters wider collaboration and increased citations

- maximum visibility for your research: over $100 \mathrm{M}$ website views per year

At $\mathrm{BMC}$, research is always in progress.

Learn more biomedcentral.com/submissions 